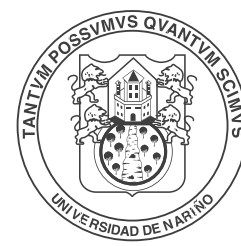

REVISTA DE CIENCIAS AGRÍCOLAS

Volumen 32(2):94 - 103

ARTICULO DE INVESTIGACIÓN

SUELOS

Segundo Semestre

ISSN Impreso 0120-0135

\title{
Efecto de la temperatura sobre coloides de suelos agrícolas mediante dispersión dinámica de la luz
}

\author{
Effect of temperature on colloids of agricultural soils through dynamic light scattering
}

Túlio Armando Lerma H. ${ }^{1}$ Enrique Miguel Combatt ${ }^{2}$; Manuel Salvador Palencia L. ${ }^{3}$

1 Tecnólogo Químico. Universidad del Valle. Cali, Colombia

2 Ph.D.Facultad de Ciencias Agrícolas, Universidad de Córdoba, Montería, Colombia.

${ }^{3}$ Ph.D. Facultad de Ciencias Naturales y Exactas, Universidad del Valle, Colombia, manuel.palencia@correounivalle.edu.co

Citar: LERMA, T.; COMBATT, 0.; PALENCIA, M. 2015. Efecto de la temperatura sobre coloides de suelos agrícolas mediante dispersión dinámica de la luz. Rev. Cienc. Agr. 32(2):94 - 103.

Fecha de recepción: Mayo 5 de 2015

Fecha de aceptación: Noviembre 11 de 2015

\section{RESUMEN}

La materia orgánica y la fracción coloidal son los principales componentes edafológicos que se ven afectados por la quema indiscriminada de los matorrales. En consecuencia, por acción de las altas temperaturas se produce un deterioro de la estructura, pérdida de la capacidad de intercambio catiónico (CIC) y disminución de la fertilidad de los suelos. Por otro lado, se ha planteado el uso de tratamiento térmico in situ como estrategia de cimentación de suelos arcillosos en la construcción de obras civiles. Sin embargo, el efecto de la temperatura sobre la fracción coloidal sólo se ha evaluado frente a propiedades mecánicas; pocos estudios en relación a propiedades de importancia agrícola como la CIC, la estructura y la susceptibilidad a la erosión han sido realizados. El objetivo del presente trabajo fue estudiar mediante dispersión dinámica de la luz (DLS) el efecto de la temperatura sobre la fracción coloidal de suelos agrícolas. Para ello se recolectaron y caracterizaron dos muestras de suelos procedentes de los departamentos de Córdoba y Valle del Cauca. La fracción coloidal fue extraída mediante una variante del método de Bouyoucos y sometida a diferentes tratamientos térmicos (desde 150 a $550^{\circ} \mathrm{C}$ ). Las muestras se caracterizaron por análisis elemental, FT-IR, DLS y se evaluó su CIC. Se concluyó que las propiedades de la fracción coloidal son fuertemente alteradas por efecto de la temperatura, además de evidenciarse una disminución de la CIC (desde 76 a 35 y desde 103 a $26 \mathrm{cmol}^{(+)} / \mathrm{kg}$ de suelo), el incremento del tamaño de partícula (desde $639 \pm 165$ a $1250 \pm 435$ y $606 \pm 102 \mathrm{a}$ $1540 \pm 320$ ) para S-Córdoba y S-Valle, respectivamente así como la eliminación de la materia orgánica.

Palabras claves: Degradación térmica, fracción coloidal, tamaño partícula, capacidad intercambio catiónico. 


\begin{abstract}
Organic matter and the colloidal fraction are the main soil components affected by indiscriminate bush burning. As a consequence, soil structure deterioration, loss of cation exchange capacity (CEC), and soil fertility reduction occur due to the high temperatures. In contrast, the use of thermal treatment has been proposed as a grounding strategy for clayey soils in civil constructions. However, the effect of temperature on the colloidal fraction has only been assessed in terms of its mechanical properties, while few studies have addressed its effect in relation to agriculturally important properties, such as CEC, structure, and erosion susceptibility. The objective of this study was to study the effect of temperature on the colloidal fraction of agricultural soils through dynamic light scattering (DLS). Two soil samples from the departments of Córdoba and Valle del Cauca were collected and characterized. The colloidal fraction was extracted through a modified Bouyoucos method and subjected to different hermal treatments (from $150^{\circ} \mathrm{C}$ to $550^{\circ} \mathrm{C}$ ). The samples were characterized according to elemental analysis, FT-IR, and DLS, and their CEC was evaluated. It was concluded that colloidal fraction properties are strongly altered by the effect of temperature. Furthermore, a decrease in CEC (from 76 to 35 and 103 to $26 \mathrm{cmol}^{(+)} / \mathrm{kg}$ soil) and an increase in particle size (from $639 \pm 165$ to $1250 \pm 435$ and 606 \pm 102 to $1540 \pm 320$ ) were observed for S-Cordoba and S-Valle, respectively; in addition to the elimination of organic matter.
\end{abstract}

Keywords: Thermal degradation, colloidal fraction, particle size, cation exchange capacity.

\section{INTRODUCCIÓN}

El suelo puede definirse como el material mineral no consolidado sobre la superficie de la tierra, que sirve como medio natural para el crecimiento de las plantas. Este está sujeto e influenciado por diversos factores formadores del suelo y el medio ambiente que son: el material parental, el clima (humedad y efectos de la temperatura), organismos y topografía (Soil Science Society of América, 2008). Sus constituyentes se pueden dividir en cuatro fracciones: inorgánica, orgánica, solución del suelo y gaseosa. La fracción inorgánica del suelo está formada por minerales, óxidos y sales. Entre estos se encuentran los minerales de arcillas, las cuales son aluminosilicatos de tipo laminar, producto de procesos de meteorización de minerales primarios, que poseen un diámetro de partícula menor a 0,002 $\mathrm{mm}$ (Bolt y Bruggenwert, 1976). Por otro lado, en la composición del suelo se encuentra la materia orgánica humificada. Esta es una mezcla heterogénea de sustancias orgánicas naturales ampliamente distribuidas en los suelos, producto de procesos biogeoquímicos (Nieder y Benbi, 2008). Estos componentes (arcillas y materia orgánica humificada) otorgan importantes propiedades de retención de agua, capacidad de intercambio catiónico (CIC), estructura, capacidad de tamponamiento, retención de elementos nutricionales y coloración, conformando junto con los óxidos metálicos lo que se conoce como la fracción coloidal del suelo (Schoonheydt y Johnston, 2013).

Por otro lado, la quema de grandes extensiones de suelo debido a causas naturales o antropogénicas, como los incendios forestales y la quema de vegetación, como una alternativa para la preparación del suelo para los cultivos, trae consigo efectos que a lo largo del tiempo resultan en actividades que empobrecen el suelo y disminuye su fertilidad natural (Dean et al., 2015). Las propiedades fisicoquímicas y biológicas se ven alteradas como resultado de las altas temperaturas alcanzadas en estos procesos y sufren alteraciones irreversibles que disminuyen irreversiblemente la fertilidad de los 
suelos. Los contenidos de nutrientes, microorganismos y materia orgánica resultan severamente alterados, siendo estas dos últimas las que poseen un mayor impacto a causa de la imposibilidad de restituir sus contenidos de forma efectiva con enmiendas agrícolas (Edivaldo et al., 2014). En este contexto, los minerales de arcilla a consecuencia del incremento de las temperaturas, sufren alteraciones y la pérdida total o parcial de su elevada superficie específica, lo que trae consigo una disminución en su reactividad superficial y consecuentemente variaciones en sus propiedades (Inbar et al., 2014).

En la actualidad la dispersión dinámica de la luz (DLS) es una técnica ampliamente utilizada en la determinación de tamaños de partícula. Su principio se basa en la dispersión de la luz generada por el movimiento browniano de las partículas en suspensión, provocando un desplazamiento en la longitud de onda, que permite determinar el coeficiente de difusión de las partículas y mediante la ecuación de Stokes-Einstein determinar el tamaño de estas (Kaszuba et al., 2008). Ya que la técnica de DLS permite monitorear los cambios de tamaño de las partículas coloidales de arcilla, surge como una herramienta útil para el estudio del efecto de la temperatura sobre algunas propiedades físicas de las arcillas que posteriormente pueden relacionarse como propiedades químicas (por ejemplo, el contenido de materia orgánica y CIC).

Esta técnica se ha empleado para evaluar el efecto de la sonicación sobre el tamaño de partícula de arcillas tipo montmorillonita evidenciándose la capacidad de la técnica para evaluar los cambios resultantes de la deslaminación de las partículas de arcillas (Poli et al., 2008), para el estudio del tamaño individual de partículas y carga aparente de la montmorillonita sódica (Cadene et al., 2005), la cinética de la coagulación de arcillas (Missana y Adell, 2000), procesos de agregación de montmorillonita y materia orgánica en ambientes que simulan el agua de mar (Furukawa et al., 2009), entre otros.

El objetivo del presente trabajo fue estudiar mediante dispersión dinámica de la luz (DLS) el efecto de la temperatura sobre la fracción coloidal de suelos agrícolas con el fin de establecer la aplicabilidad de la técnica de DLS en la evaluación del impacto de las quemas, de origen natural 0 antropogénico de los suelos.

\section{MATERIALES Y MÉTODOS}

Caracterización de las muestras de suelo. Dos suelos diferentes se recolectaron en los departamentos de Córdoba y Valle del Cauca (Colombia). Previo a los análisis de caracterización, cada muestra de suelo se secó a temperatura ambiente, se molió y tamizó empleando un tamiz de $2 \mathrm{~mm}$. La caracterización de los suelos se efectuó en las instalaciones del Laboratorio de Suelos y Agua de la Universidad de Córdoba (Montería-Colombia). A las muestras se les determinó la textura, $\mathrm{pH}$, bases intercambiables ( $\mathrm{Na}, \mathrm{K}, \mathrm{Ca}$ y $\mathrm{Mg}$ ) y contenido de materia orgánica (IGAC, 2006). Los ensayos fueron realizados por triplicado identificando los suelos como S-Córdoba y S-Valle en concordancia con el lugar de procedencia respectivo.

Extracción de fracción coloidal. La extracción de la fracción arcillosa de las muestras de suelo se realizó mediante una variante del método de Bouyoucos. Para ello, $60 \mathrm{~g}$ de suelo se dispersaron en agua, utilizando una solución de hexametafosfato de sodio $\left(\left(\mathrm{NaPO}_{3}\right)_{6} 96 \%\right.$, Aldrich $)$ y carbonato de sodio $\left(\mathrm{Na}_{2} \mathrm{CO}_{3}, 99 \%\right.$, Aldrich como agentes dispersantes. La dispersión coloidal se dejó con agitación constante a temperatura ambiente por doce horas. Posteriormente, la dispersión se llevó a una probeta con capacidad de1 L, se alcanzó el aforo con agua destilada, y se llevó a cabo su homogenización mediante agitación 
mecánica para posteriormente dejarse en reposo por $2 \mathrm{~h}$. Finalmente, se extrajo el sobrenadante y se sometió a centrifugación por 15 min a 5000 rpm. Las arcillas se secaron a temperatura ambiente y se caracterizaron por análisis elemental (FlashEA 1112 Automatic Elemental Analyzers), espectroscopia infrarroja transformada de Fourier mediante la técnica de Reflactancia Total Atenuada (FT-IR/ATR; IRAffinity-1), DLS (Malvern, Zetasizer nano zs) y se determinó su CIC por el método acetato de amonio $1 \mathrm{~N}$ a pH 7 (IGAC, 2006).

Estudio degradación térmica. El estudio de degradación térmica se llevó a cabo mediante calentamiento a diferentes temperaturas. Para esto, $10 \mathrm{~g}$ de la fracción coloidal obtenidas en la etapa previa se sometió a calentamiento por 4 horas. El procedimiento se realizó a $150,250,350,450$ y $550{ }^{\circ} \mathrm{C}$ para cada una de las dos fracciones extraídas (SCórdoba y S-Valle). La Montmorrillonita (Aldrich) se utilizó como blanco, sometiéndose $10 \mathrm{~g}$ de esta al mismo procedimiento térmico indicado previamente. Las muestras se caracterizaron por análisis elemental, DLS y se determinó su CIC (IGAC, 2006).

\section{RESULTADOS Y DISCUSIÓN}

Caracterización de las muestras de suelo. Los resultados de la caracterización de los suelos se detallan en la Tabla 1. Como se puede observar, el pH para el S-Córdoba es ligeramente ácido y para el S-Valle ligeramente alcalino. Los suelos seleccionados poseen una textura fina, lo cual permite obtener altos rendimientos en el proceso de extracción de la fracción coloidal (arcillosa y franco arcillosa para S-Córdoba y S-Valle, respectivamente). Los contenidos de materia orgánica fueron medios para S-Córdoba y deficientes para S-Valle, sugiriendo una baja contribución de la materia orgánica sobre la CIC. Los contenidos de bases intercambiables en ambos suelos fueron relativamente altos, indicativo de la alta fertilidad de los suelos. Por otro lado, ambos suelos presentaron una capacidad de intercambio catiónico efectiva (CICe) alta, lo cual puede atribuirse a los altos contenidos de arcilla y, en el caso de S-Córdoba, también a los contenidos relativamente moderados de materia orgánica para S-córdoba, lo cual se ve reflejado en los resultados de análisis textural.

Tabla 1. Análisis de caracterización agrícola de la muestras de suelo.

\begin{tabular}{lccc}
\hline Parámetro & Unidad & S-Córdoba & S-Valle \\
\hline Textura & --- & Arcilloso & Franco-Arcilloso \\
$\mathrm{pH}$ & $1: 1$ & 6,74 & 7,47 \\
$\mathrm{MO}$ & $\%$ & $2,73(\mathrm{c})$ & $0,97(\mathrm{~d})$ \\
$\mathrm{Ca}^{2+}$ & $\mathrm{cmol}^{(+)} / \mathrm{kg}$ & $47,5(\mathrm{a})$ & $77,74(\mathrm{a})$ \\
$\mathrm{Mg}^{2+}$ & $\mathrm{cmol}^{(+)} / \mathrm{kg}$ & $7,2(\mathrm{a})$ & $32,73(\mathrm{a})$ \\
$\mathrm{Na}^{+}$ & $\mathrm{cmol}^{(+)} / \mathrm{kg}$ & $0,42(\mathrm{a})$ & $0,44(\mathrm{a})$ \\
$\mathrm{K}^{+}$ & $\mathrm{cmol}^{(+)} / \mathrm{kg}$ & $0,98(\mathrm{a})$ & $1,92(\mathrm{a})$ \\
$\mathrm{CICe}$ & $\mathrm{cmol}^{(+)} / \mathrm{kg}$ & 58,3 & 30,91 \\
\hline
\end{tabular}

Diagnóstico del suelo:(a) contenido abundante o valor alto pero no excesivo, (b) contenido suficiente o valor adecuado, (c) contenido moderado o valor medio y (d) contenido deficiente o valor bajo. MO: Materia orgánica. CICe: Capacidad de intercambio catiónico efectiva. 
Extracción de la fracción coloidal. La fracción coloidal de los suelos pudo ser extraída apropiadamente mediante la variante del método de Bouyoucos descrita previamente. En la Figura 1 se muestra el análisis por FT-IR/ATR de las fracciones obtenidas. La banda principal en torno a $3650 \mathrm{~cm}^{-1}$ corresponde a la vibración de tensión de los grupos -OH enlazados a los cationes de la capa octaédrica, si bien la posición de esta banda está relacionada con la composición química de la capa octaédrica y puede aparecer en posiciones distintas en cada mineral, se ha reportado que la presencia de cationes trivalentes como el $\mathrm{Al}^{3+}$ y el Fe${ }^{3+}$ desplazan la banda a frecuencias menores, mientras que la presencia de cationes divalentes como el $\mathrm{Mg}^{2+}$ desplaza la posición de la banda a frecuencias más elevadas (Camejo, 2013).

De acuerdo con los análisis de caracterización, los valores de $\mathrm{pH}(>5,5)$ sugieren que no existe liberación de $\mathrm{Al}^{3+}$ en los suelos estudiados, por lo cual es de esperarse un desplazamiento de la banda como resultado de la adsorción de $\mathrm{Mg}^{2+}$ y $\mathrm{Ca}^{2+}$ principalmente. En S-Córdoba los contenidos de $\mathrm{Mg}^{2+}<\mathrm{Ca}^{2+}$, sugiriendo esto que la carga del ión más que su naturaleza promueve el desplazamiento de esta señal en las regiones observadas.

A $3400 \mathrm{~cm}^{-1}$ aparece una banda más ancha correspondiente a las vibraciones de los grupos -OH que participan en enlaces de hidrógeno de las moléculas de agua ubicadas en el espacio interlaminar. Además, en torno a $1600 \mathrm{~cm}^{-1}$ se observó una banda asociada a la vibración de flexión de los grupos - $\mathrm{OH}$ también relacionada con el $\mathrm{H}_{2} \mathrm{O}$ del espacio interlaminar. Como estas bandas se encuentran presentes en tanto en la montmorillonita como en los minerales extraídos de cada uno de los suelos, se demuestra sin duda la presencia de agua en los mismos. Esto es congruente con lo reportado por otros trabajos (Camejo, 2013; Villegas, 2013).

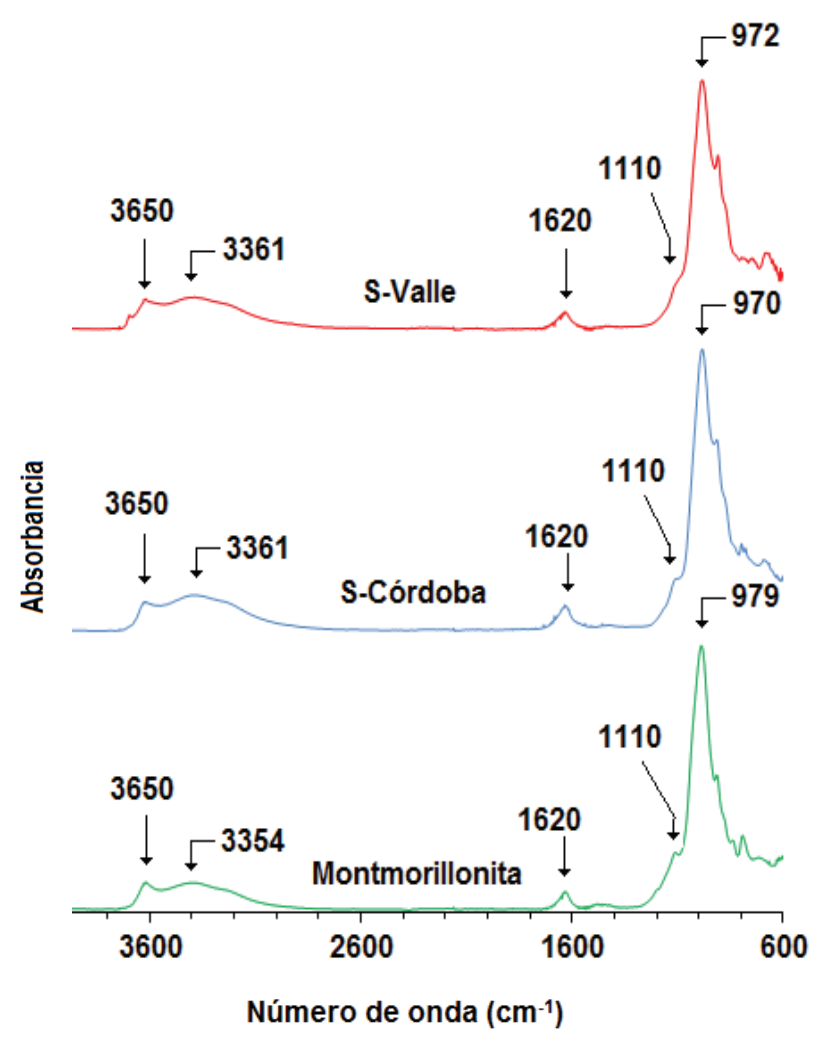

Figura 1. Espectro FT-IR/ATR de las fracciones coloidales obtenidas.

En la región entre 600 y $1200 \mathrm{~cm}^{-1}$ aparecen bandas características de las esmectitas (mineral tipo silicato cuyo principal representante es la montmorillonita). La banda más intensa alrededor de $1000 \mathrm{~cm}^{-1}$, es característica de la vibración de tensión del enlace Si-O-Si de la capa tetraédrica de las esmectitas. La aparición de una pequeña señal aproximadamente en $1110 \mathrm{~cm}^{-1}$ se atribuye a la sustitución isomorfica de átomos de silicio por átomos de aluminio en la capa tetraédrica. A frecuencias mayores, es decir, entre número de ondas entre $600-920 \mathrm{~cm}^{-1}$ aparecen generalmente un conjunto de bandas de baja intensidad asociadas con vibraciones de flexión de los enlaces que constituyen las capas del mineral (Camejo, 2013).

La similitud de los perfiles de los espectro de absorción de las muestras en relación al de la montmorillonita, indican que la composición principal de las fracciones coloidales obtenidas son 
de origen mineral para ambos suelos (Madejová et al., 2010).

En la Tabla 2 se muestran los contenidos de carbono, nitrógeno e hidrógeno medidos por análisis elemental, tamaño promedio de partícula y la CIC de las muestras. Las propiedades coloidales de las fracciones se evidencian por poseer propiedades características de las arcillas, tamaños de partículas inferiores a 0,002 $\mathrm{mm}$ y su elevada capacidad de CIC de 76 y $103 \mathrm{cmol}^{(+)} / \mathrm{kg}$ para S-Córdoba y $\mathrm{S}$-Valle respectivamente.

Por otro lado, los bajos contenidos de los elementos medidos pueden relacionarse con las relativamente bajas concentraciones de materia orgánica en los suelos. Además, en los suelos la materia orgánica humificada y las arcillas se encuentran íntimamente relacionadas mediante mecanismos de interacción de fuerzas de Van der Waals, intercambio iónico, enlace de hidrógeno e intercambio de ligando. Siendo la humina la de mayor interacción y prevalencia en la fracción extraída, viéndose entonces disminuidos los contenidos de los elemento en el proceso de extracción.

Estudio degradación térmica. En la Figura 2 se muestra la pérdida de masa debido al calentamiento de las fracciones coloidales. Se puede observar que la cantidad de masa disminuye al incrementar la temperatura para todas las muestras. Esta variación puede explicarse debido a la pérdida de agua en forma de vapor y la oxidación de la materia orgánica presente en la muestra.

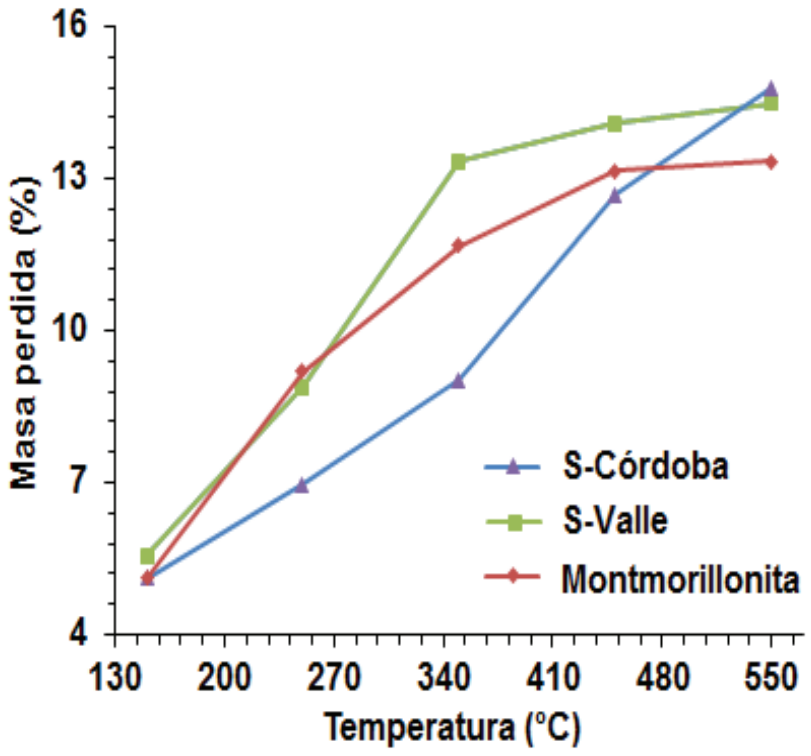

Figura 2. Masa perdida en función de la temperatura.

En la Figura 3 se muestra la pérdida de elementos con el incremento de la temperatura. Se puede observar la disminución de los contenidos de elementos como carbono, nitrógeno e hidrógeno provenientes de la materia orgánica del suelo producido por el incremento de la temperatura que causa reacciones de oxidación, convirtiendo la materia orgánica presente en gases que son liberados a la atmósfera y la pérdida total o parcial de sus contenidos, generando cambios en las propiedades físicas y químicas de los suelos. Además, como se observa el nitrógeno por encima de temperaturas de los $350{ }^{\circ} \mathrm{C}$ puede llegar a estar ausente y este al ser uno de los nutrientes básicos para las plantas, al disminuir considerablemente sus contenidos se esperan alteraciones de la fertilidad de los suelos.

Tabla 2. Diámetros de partícula, CIC y elementos medidos.

\begin{tabular}{lccccc}
\hline Muestra & Diámetro & CIC & \multicolumn{3}{c}{ Análisis elemental (\%) } \\
& partícula (nm) & $\left.\mathbf{c m o l}^{(+)} / \mathbf{k g}\right)$ & Carbono & Nitrógeno & Hidrógeno \\
\hline S-Córdoba & $645 \pm 184$ & 76 & 0,95 & 0,29 & 1,38 \\
S-Valle & $445 \pm 80$ & 103 & 1,07 & 0,11 & 1,81 \\
Montmorillonita & $974 \pm 346$ & 60 & 0,29 & 0 & 1,21 \\
\hline
\end{tabular}




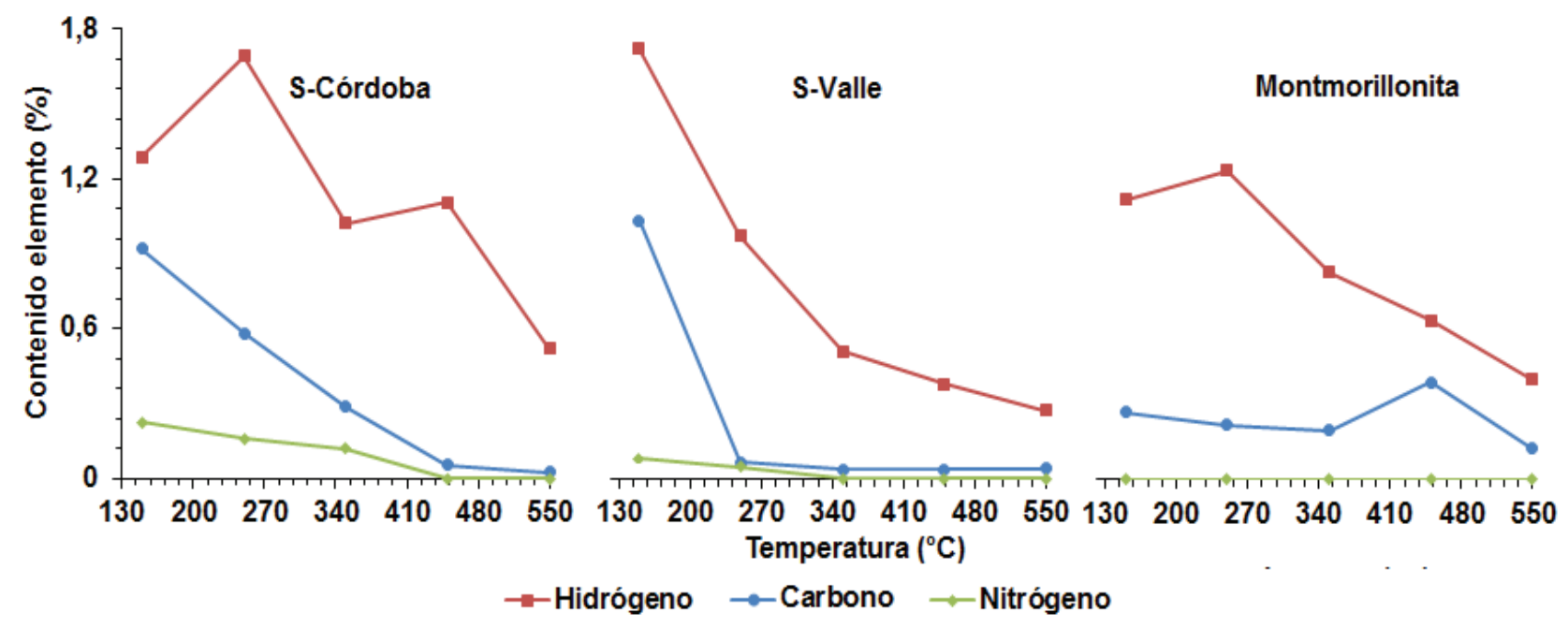

Figura 3. Contenido de elementos medidos en función de la temperatura.

El incremento de la temperatura genera pérdidas considerable en la CIC de la fracción coloidal de hasta el 70 \% (S-Valle), como se observa en la Figura 4. Esta disminución se pude explicar debido a los cambios físicos y químicos que experimenta la fracción coloidal. A causa, de la pérdida de la materia orgánica por oxidación térmica, la cual en suelos con contenidos significativamente altos, representará importantes cambios en su CIC. Además, los cambios producto de las altas temperatura en la estructura de los minerales de arcilla a causa de la eliminación del agua adsorbida y la deshidroxilación superficial y estructu-

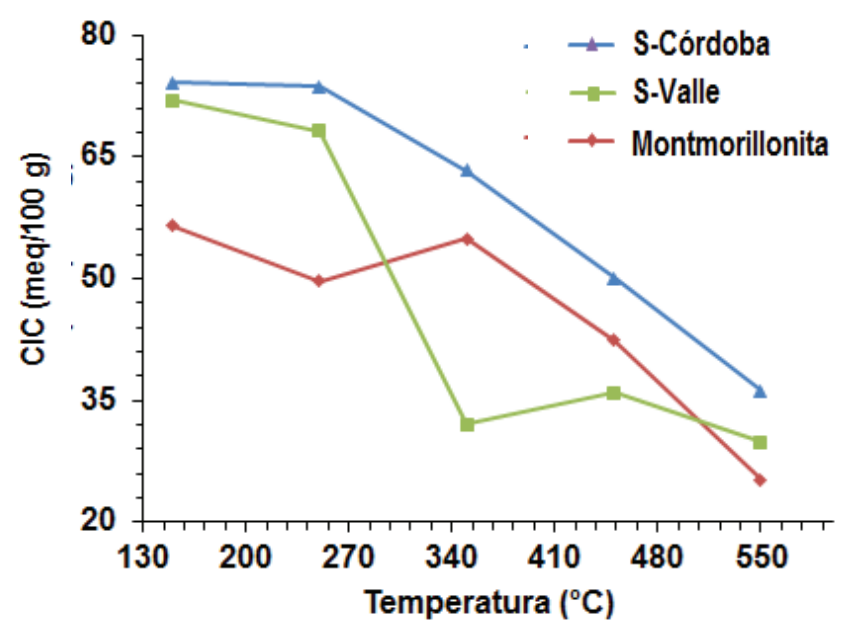

Figura 4. CIC de la fracción coloidal de los suelos en función de la temperatura. ral, genera la pérdida de sus propiedades (CIC, retención agua) y la imposibilidad de su regeneración natural (Verma y Jayakumar, 2012).

Por otro lado, para determinar el tamaño de las partículas mediante la técnica de DLS no se tiene en cuenta la dependencia de la intensidad de la luz dispersada respecto al ángulo de dispersión, sino la variación de la intensidad de dispersión en el tiempo. En esta técnica la luz láser al interactuar con las partículas que hay en una suspensión, se dispersa en todas las direcciones posibles. Como consecuencia del movimiento browniano las posiciones relativas de las partículas varían constantemente entre sí, cosa que también provoca cambios en las condiciones de interferencia y en la propia intensidad de dispersión. Si las partículas se mueven rápidamente (partículas pequeñas), también se acelera la variación de la intensidad de dispersión. Por el contrario, las partículas lentas (grandes) llevan a variaciones más lentas. Así, si frente a un determinado tratamiento térmico, si los tiempos de relajación de la luz dispersada varían estos estarán asociados a procesos de agregación que cambian la velocidad de desplazamiento de las partículas de arcilla en el medio. En consecuencia, un aumento o disminución del tamaño puede ser correlacionado con los tratamientos térmicos y de esta forma obtenerse información sobre los cambios experimentados por las partículas coloidales. 
La Figura 5 muestra los tamaños promedio de partícula medidos por DLS de las muestras sometidas a diferentes temperaturas. Se evidencia un incremento del tamaño de partícula a causa de las temperaturas, generando cambios estructurales sobre los suelos. Este fenómeno ocasiona la disminución de la superficie específica de los minerales de arcilla a causa de la deshidrogenación superficial generada por las altas temperaturas que ocasionará nuevos enlaces entre los coloides de arcilla e incrementar su tamaño (Verma y Jayakumar, 2012). A partir de los $450^{\circ} \mathrm{C}$ se inicia la pérdida de las propiedades coloidales dando como resultado su precipitación total (Tabla 3).

Ya que la técnica de DLS se basa en el movimiento browniano de las partículas en suspensión y los nuevos agregados no se encuentran regidos bajo

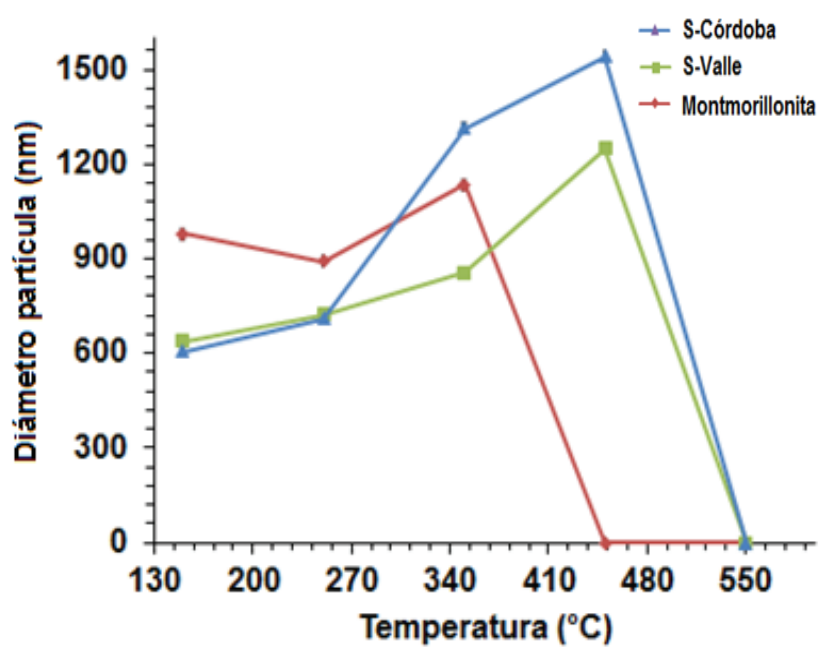

Figura 5. Diámetro de partícula en función de la temperatura. este principio, no es posible la determinación de su tamaño, lo que sugiere que ha tenido lugar la pérdida total de sus propiedades coloidales. Repercutiendo no solo en las propiedades físicas del suelo, sino también en sus propiedades químicas de CIC, pH y contenidos de nutrientes.

Por otra parte, se ha indicado que la textura del suelo es un factor que define la magnitud de las modificaciones que experimenta un suelo ante la exposición a altas temperaturas. El efecto principal se ha reportado sobre la fracción de arena y limo respecto a un cambio menos marcado en la fracción arcillosa (Simeon, 2012).

Los resultados obtenidos son congruentes con lo reportado por Villegas (2013) mediante el análisis termogravimétrico de la montmorillonita. Así, se ha indicado una pérdida de peso en el intervalo comprendido entre $20-90{ }^{\circ} \mathrm{C}$ que corresponde a la eliminación del agua fisiadsorbida entre las láminas se silicato. Además, una segunda perdida en masa en un intervalo comprendido entre $150-440^{\circ} \mathrm{C}$ que corresponde a la fácil remoción de los grupos hidroxilos de los extremos de las láminas de arcillas, los cuales son asociados a la estructura interna de las láminas. Finalmente, la tercera pérdida de peso sobre los $490-700^{\circ} \mathrm{C}$ que corresponde a una deshidroxilación total de la estructura arcillosa; mostrando a su vez el comienzo de una posible reorientación de dicha estructura que puede dar origen a un colapso a temperaturas superiores (Villegas, 2013).

Tabla 3. Diámetros de partícula en función de la temperatura.

\begin{tabular}{lccccc}
\hline Temperaturas $\left({ }^{\circ} \mathrm{C}\right)$ & 150 & 250 & 350 & 450 & 550 \\
S-Córdoba & $639 \pm 165$ & $723 \pm 154$ & $857 \pm 90$ & $1250 \pm 435$ & -- \\
S-Valle & $606 \pm 102$ & $709 \pm 131$ & $1313 \pm 598$ & $1540 \pm 320$ & --- \\
Montmorillonita & $978 \pm 90$ & $893 \pm 234$ & $1134 \pm 143$ & --- & --- \\
\hline
\end{tabular}


Los resultados indican que el efecto de la temperatura sobre la fracción arcillosa sólo es significativo para temperaturas superiores a $150{ }^{\circ} \mathrm{C}$, para los tiempos de exposición evaluados. Aunque esto sugiere un intervalo de efecto mínimo, la exposición a temperaturas mayores es posible en situaciones relativamente habituales como los incendios forestales o prácticas agrícolas inadecuadas como el acondicionamiento del terreno mediante la quema programada. En este sentido, se ha reportado mediante estudios hechos con cámaras termográficas que las temperaturas, en el caso de incendios a pequeña escala, pueden alcanzar valores entre 207 y $237^{\circ} \mathrm{C}$ (valor medio $237^{\circ} \mathrm{C}$ ). Sin embargo, para quema prescrita, las temperaturas máximas pueden estar comprendidas entre 768 y $1118^{\circ} \mathrm{C}$, con los mínimos de temperatura en el extremo superior de la llama (Zarate, 2004; Reynard-Callanan et al., 2010). En consecuencia, es de esperarse que frente a procedimientos de quema las partículas coloidales del suelo se expongan a temperatura tales que las propiedades coloidales de los suelos se alteren completamente.

\section{CONCLUSIONES}

Las propiedades de la fracción coloidal del suelo son alteradas por las elevadas temperaturas, generando la eliminación de la fracción orgánica y modificación del material mineral. Los contenidos de elementos como carbono, hidrogeno y nitrógeno, en especial este último disminuye hasta su total eliminación, generando una pérdida en la fertilidad del suelo. La CIC se disminuye hasta en un $70 \%$ como producto de la perdida de la materia orgánica y alteraciones estructurales de las arcillas. Los diámetros de partícula de las arcillas aumentan como consecuencia de la condensación de las partículas con la consecuente variación de su superficie especifica. Por último, los resultados indican que a temperaturas superiores a $150^{\circ} \mathrm{C}$ las propiedades coloidales de las arcillas se ven afectadas.

\section{REFERENCIAS BIBLIOGRÁFICAS}

BOLT, G. y BRUGGENWERT, M. 1976. Chapter 1: Composition of the Soil. In Soil. Developments in Soil Science. $5(\mathrm{~A}): 1$ - 12.

CADENE, A., DURAND-VIDAL, S.,TURG, P. y BRENDLE, J. 2005. Study of individual Na-montmorillonite particles size, morphology, and apparent charge. Journal of Colloid and Interface Science. 285:719 - 730.

CAMEJO, C. 2013. Modificación de arcillas comerciales y naturales para el diseño de nuevos sistemas catalíticos. Tesis doctoral. Universidad de Alcalá. Alcalá - España. 279 p.

DEAN, S., FARRER, E. y MENGES, E. 2015. Fire Effects on Soil Biogeo Chemistry in Florida Scrubby Flatwoods. American Midland Naturalist. 174(1):49.

EDIVALDO, L.,THOMAZ, V. y STEFAN H. 2014. Effects of fire on the physicochemical properties of soil in a slashand-burn agriculture.CATENA. 122:209 - 215.

FURUKAWA, Y., WATKINS, J., KIM, J., CURRY, K. y BENNETT, R. 2009. Aggregation of montmorillonite and organic matter in aqueous media containing artificial seawater. Geochemical Transaction. 10:1 - 11.

INBAR, A., LADO, M., STERNBERG, M.;TENAU, H. y BENHUR, M. 2014. Forest fire effects on soil chemical and physicochemical properties, infiltration, runoff, and erosion in a semiarid Mediterranean region. Geoderma. 221 - 222:131 - 138.

INSTITUTO GEOGRÁFICO AGUSTÍN CODAZZI (IGAC). 2006. Métodos analíticos delaboratorio de suelos. VI Edición. Bogotá, Subdirección de Agrología.

KASZUBA, M., MCKNIGHT, D.,CONNAH, M., MCNEILWATSON, F. y NOBBMANN, U. 2008. Measuring sub nanometre sizes using dynamic light scattering. Journal of Nanoparticle Research. 10(5):823 - 829.

MADEJOVÁ, J., PÁLKOVÁ, H. y KOMADEL, P. 2010. IR spectroscopy of clay minerals and clay nanocomposites.Spectroscopic Properties of Inorganic and Organometallic Compounds: Techniques, Materials and Applications. 41:22 - 71 . 
MISSANA, T. y ADELL, A. 2000. On the Applicability of DLVO Theory to the Prediction of Clay Colloids Stability. Journal of Colloid and Interface Science, 230:150 - 156.

NIEDER, R. y BENBI, D. 2008. Carbon and Nitrogen in the Terrestrial Environment. Springer Netherlands, Dordrecht. 81 - 111.

POLI, A., BATISTA, T., SCHMITT, C., GESSNER, F. y NEUMANN, M. 2008. Effect of sonication on the particle size of montmorillonite clays. Journal of Colloid Interface Science 325(2):386 - 390.

REYNARD-CALLANAN, J., POPE, G.,GORRING, M. y FENG, H. 2010. Effects of high-intensity forest fires on soil clay mineralogy. Physical Geography, 31:407 - 422.

SCHOONHEYDT, R. y JOHNSTON, C. 2013. Chapter 5 Surface and Interface Chemistry of Clay Minerals. En: Bergaya, F. y Lagaly, G.(Ed). Handbook of Clay Science; Science, Elsevier, 139 - 172.

SIMEON, B. 2012. Forest fire influence on soil texture in burned forests in Bulgaria. Forestry Ideas. 18(2):155 - 162.

SOIL SCIENCE SOCIETY OF AMERICA. 2008. Glossary of Soil Science Terms. Soil Science Society of America, Wisconsin. $57 \mathrm{p}$.

VERMA, S. y JAYAKUMAR, S. 2012. Impact of forest fire on physical, chemical and biological properties of soil: Areview. Proceedings of the International Academy of Ecology and Environmental Sciences, 2(3):168 - 176.

VILLEGAS, E. 2013. Modificación y caracterización de un material arcilloso tipo esmectita de potencial aplicación en catálisis. Tesis de Magister. Universidad Nacional de Colombia. Medellin. 67 p.

ZARATE, L. 2004. Estudio de la temperatura de la llama en incendios forestales. Tesis doctoral. Universitat Politècnica de Catalunya. 